\title{
Microscopic pulmonary neoplastic emboli: report of a case with respiratory failure but normal imaging
}

\author{
Jose Miguel Chatkin*, Leandro Genehr Fritscher, Jussara Fiterman, \\ Carlos Cezar Fritscher, Vinicius Duval da Silva
}

Hospital São Lucas da PUCRS, Porto Alegre, Brazil

Received 24th August 2006; accepted 18th December 2006

\author{
KEYWORDS \\ Neoplastic circulating \\ cells; \\ Pulmonary embolism; \\ Respiratory \\ insufficiency; \\ Adenocarcinoma; \\ Breast neoplasm
}

\begin{abstract}
Pulmonary tumour microembolism is a recognised cause of respiratory failure in patients with cancer, but is frequently under-recognised. We report the case of a 42-year-old female patient admitted because of rapidly progressive severe dyspnoea with a normal chest X-ray (CXR). Initial investigation and imaging was inconclusive, with normal computerised tomography (CT) scans and pulmonary angiotomography. Videothoracoscopic biopsy was performed. Microscopic examination revealed metastatic adenocarcinoma in the lungs, with pulmonary vasculature showing numerous microemboli in small arterioles. The site of origin was consistent with a primary breast adenocarcinoma resected three years previously. Despite treatment in the Intensive Care Unit, the patient died 72 hours after admission. Active consideration and investigation for malignancy should always be considered in the differential diagnosis of patients with severe unexplained dyspnoea.

(c) 2007 General Practice Airways Group. All rights reserved.
\end{abstract}

\section{Introduction}

Pulmonary tumour embolism (PTE) is not an uncommon cause of respiratory failure in patients with cancer, but it is rarely recognised antemortem. ${ }^{1,2}$ Although the real frequency is not fully known, it is reported to be present in up to $26 \%$ of all cancer autopsies. ${ }^{2}$ PTE without any significant parenchymal metastases was seen in only $2.4 \%$ of cases. ${ }^{3}$ We describe the case of a patient presenting

* Corresponding author.

Hospital São Lucas da PUCRS, Av. Ipiranga 6690, 3rd.floor, 90610-000 Porto, Alegre, BRAZIL

Tel: +55 513320 3378; fax: +55 5133203316

E-mail address: jmchatkin@pucrs.br with fulminant respiratory failure, with normal initial imaging but progression to death in 72 hours, which was due to pulmonary tumour embolism.

\section{Case report}

A 42-year-old female presented with progressive dyspnoea of four weeks' duration without any other respiratory symptoms, worsening over the last couple of days. She had had excision of a breast adenocarcinoma three years previously. On admission, she was in respiratory distress (respiratory rate $28 / \mathrm{min}$ ) with mild cyanosis, and pulse was 110 beats/minute. Weight was $65 \mathrm{~kg}$, height $168 \mathrm{~cm}$, lungs were clear on auscultation and 


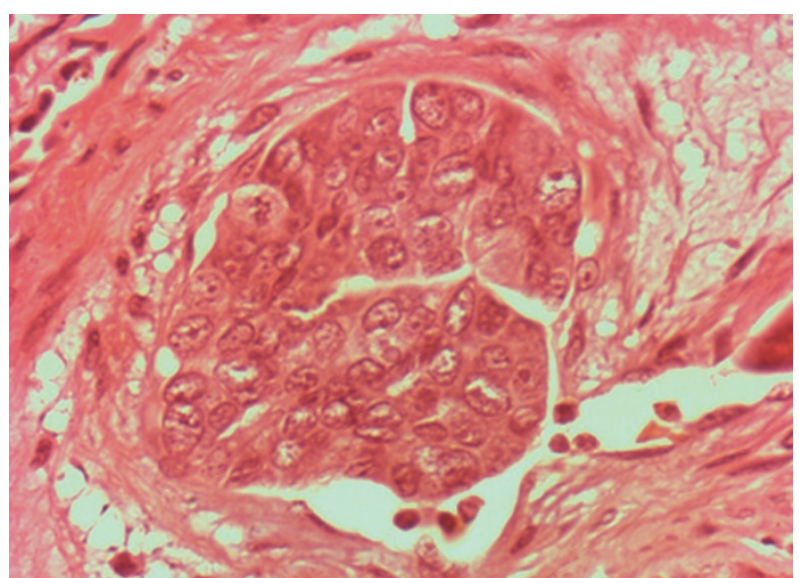

Figure 1 Neoplastic cells inside a blood vessel showing chromatin alterations and pleomorphic nuclei and cell size variations. H\&E, 400X.

there was a slightly accentuated pulmonary valve component on the second heart sound. Examination of the breasts revealed the previous partial right mastectomy but nothing else.

Biochemical and haematological analysis, including liver and thyroid function tests, cardiac enzymes, connective tissue disease markers and full blood profile were normal. Blood gas analysis showed a pH of $7.31, \mathrm{pO}_{2} 48 \mathrm{mmHg}, \mathrm{pCO}_{2} 32 \mathrm{mmHg}$, oxygen saturation of $86 \%$, and $\mathrm{HCO}_{3} 27 \mathrm{mEq} / \mathrm{l}$. Chest $X$-ray (CXR) showed diminished right breast volume with otherwise normal pulmonary parenchyma. Electrocardiogram showed only sinus tachycardia. Echocardiography demonstrated preserved systolic function and normal function on the right heart side. Estimated pulmonary artery systolic pressure was $56 \mathrm{mmHg}$, with normal left ventricular ejection fraction. Pulmonary function tests and lung volumes were normal. Diffusing capacity of carbon monoxide (DLCO) was moderately reduced (52\%).

Chest and abdominal computerised tomography (CT) scans were reported as normal. Pulmonary angiotomogram was also normal.

Due to the rapidly worsening dyspnoea, pulmonary arteriography was performed in order to exclude pulmonary embolism. Vascular perfusion was normal and the pulmonary artery systolic pressure was $40 \mathrm{mmHg}$.

The patient was then submitted to videothoracoscopic lung biopsy. Over the next 12 hours the patient's oxygenation levels deteriorated further and she required intubation and mechanical ventilation. Hypoxia worsened, accompanied with hypotension unresponsive to inotropes, and the patient died 72 hours after admission.

Microscopic examination revealed metastatic adenocarcinoma in the lungs, with pulmonary vasculature showing numerous microemboli in small

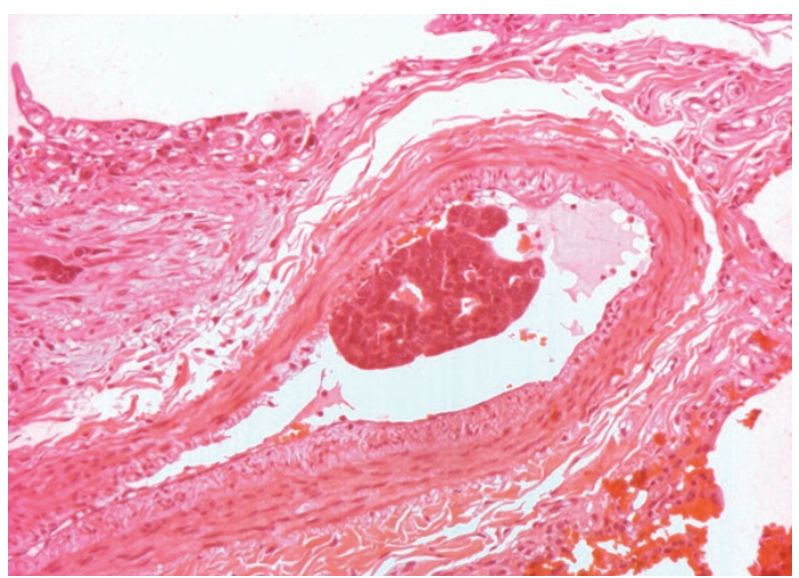

Figure 2 Artery showing intraluminal neoplastic embolus. H\&E, 100X.

arterioles. The site of origin was consistent with a primary breast adenocarcinoma (Figures 1 and 2).

\section{Discussion}

This patient had progressive sub-acute dyspnoea with a rapid stepwise progression and fulminant presentation. She had remained very well until three days before her death. The fulminant progression in cases like this has been attributed to recurrent showers of tumour microemboli and changes in the pulmonary vascular bed. ${ }^{1}$

There was a broad differential diagnosis when the patient first presented to us. ${ }^{4}$ One of our first thoughts - in a young female with dyspnoea, severe hypoxemia and clear lungs on examination was pulmonary embolism, a condition associated with malignant lesions. After normal angiotomography, we submitted the patient to pulmonary arteriography, looking for more peripheral emboli. This showed no evidence of embolism or other abnormalities. An echocardiogram showed a modest increase in pulmonary pressures without right or left ventricular dysfunction.

These procedures also helped us to exclude veno-arterial shunts and disorders of the precapillary pulmonary vascular bed. Extrapulmonary veno-arterial shunts, usually secondary to cirrhosis of the liver, were also excluded by the normal liver enzymes, and the clinical picture for these conditions is rarely severe enough to produce the symptoms and signs that our patient had.

Lymphangitic carcinomatosis was a possibility; this is a common complication of breast cancer which can present with dyspnoea and a normal CXR, but the diagnosis was excluded after the normal chest CT scan. 
Normal pulmonary function tests with normal volumes and decreased DLCO is a situation that can occur in a variety of interstitial or vascular disorders. Systemic lupus erythematosis and scleroderma were also initially considered, but they were excluded by the normal rheumatological examination and the absence of other clinical evidence of a collagen disorder. Usually, such diseases can cause insidious cor pulmonale, in contrast to the short duration of symptoms in this case.

Although considered in the preliminary investigations, acute polyneuritis and myasthenia-like syndromes were also excluded.

The decision to proceed to a pulmonary biopsy was based on the progressive and rapid clinical deterioration. This procedure confirmed the hypothesis of microscopic tumour embolisation.

Sub-acute progressive dyspnoea is the most common initial clinical symptom of microscopic pulmonary tumour embolism. ${ }^{5}$ Systemic symptoms such as fatigue and weight loss are infrequently present. Physical examination usually demonstrates signs of pulmonary hypertension in association with tachypnoea, tachycardia, unremarkable findings on lung auscultation and an accentuated pulmonary valve component on the second heart sound. The initial laboratory evaluation usually shows hypoxemia with a normal CXR.

PTE was first reported by Brill and Robertson in $1937,{ }^{6}$ who described a case of rapid development of right heart failure due to tumour emboli. PTE is characterised by occlusion of small pulmonary arteries, arterioles and alveolar septal capillaries by aggregates of tumour cells accompanied by platelet-fibrin thrombosis. Pulmonary complaints may be the initial presentation of a neoplasia or a terminal event. When the cancer is apparently under remission, the diagnosis is less clear and sometimes forgotten. In a recent report of 43 cases of pulmonary infarction, only one was secondary to malignancy. ${ }^{7}$

The overall survival is usually four to 12 weeks in the very few patients who have a diagnosis made prior to death. ${ }^{8}$
This case underlines the diagnostic difficulties in managing PTE. ${ }^{9}$ It demonstrates the need to consider the possibility of a diagnosis of PTE in cancer patients who present with non-explained dyspnoea, ${ }^{10}$ many of whom will present in primary care.

\section{Conflict of interest declaration}

There were no conflicts of interest for the authors during the preparation of this manuscript.

\section{References}

1. Hibbert $M$, Braude $S$. Tumour microembolism presenting as “primary pulmonary hypertension". Thorax 1997;52(11): 1016-17.

2. Schriner RW, Ryu JH, Edwards WD. Microscopic pulmonary tumor embolism causing subacute cor pulmonale: a difficult antemortem diagnosis. Mayo Clin Proc 1991; 66(2):143-8.

3. Kane RD, Hawkins HK, Miller JA et al. Microscopic pulmonary emboli associated with dyspnoea. Cancer 1975; 36:1473-82.

4. Respiratory disease associated with a normal chest roentgenogram. In: Fraser RG, Paré JAP, Pare PD et al, editors. Diagnosis of diseases of the chest. Philadelphia, USA: WB Saunders Co, 2005: 2974-3012.

5. Geschwind JF, Dagli MS, Vogel-Claussen J et al. Metastatic breast carcinoma presenting as a large pulmonary embolus: case report and review of the literature. $\mathrm{Am} \mathrm{J}$ Clin Oncol 2003;26(1):89-91.

6. Brill IC, Robertson TD. Subacute cor pulmonale. Arch Intern Med 1937;60:1043-57.

7. Parambil JG, Savci CD, Tazelaar HD et al. Causes and presenting features of pulmonary infarctions in 43 cases identified by surgical lung biopsy. Chest 2005;127(4): 1178-83.

8. Gonzales-Vitale JC, Garcia-Bunel R. Pulmonary tumour emboli and cor pulmonale in primary carcinoma of the lung. Cancer 1976;38:2105-10.

9. Steiner S, Plehn G, Reinecke P et al. Disseminated microvascular pulmonary tumor cell embolism: a rare cause of fulminant pulmonary hypertension. Onkologie 2004;27(6):566-8.

10. Systrom DM, Mark EJ. Weekly Clinicopathological Exercises: Case 19-1995: A 55-Year-Old Woman with Acute Respiratory Failure and Radiographically Clear Lungs. N Engl J Med 1995;332(25):1700-07.

Available online at http://www.thepcrj.org 